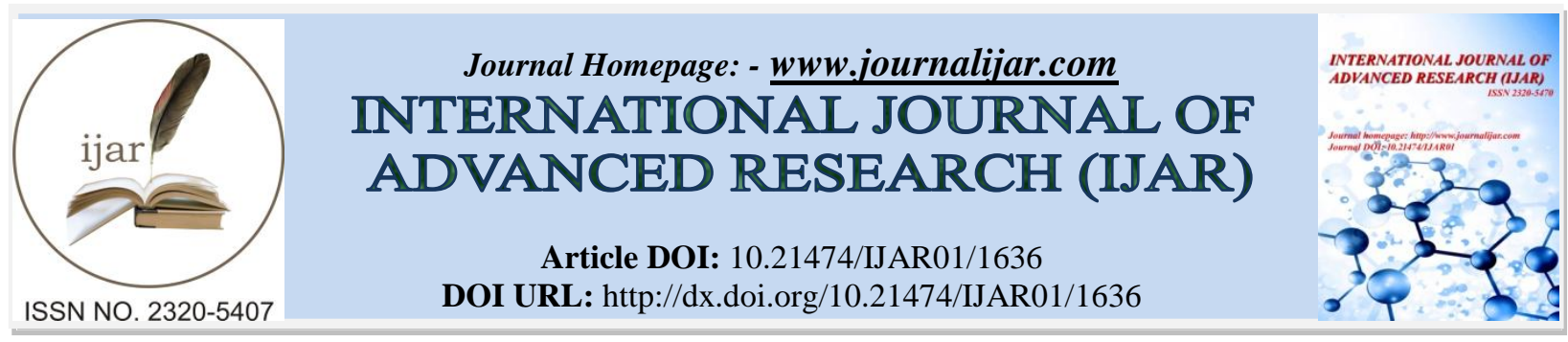

RESEARCH ARTICLE

\title{
First identification of Trichophyton rubrum var. raubitschekii in Constantine (ALGERIA).
}

\section{Anissa Zohra Hafirassou ${ }^{1}$, Nadia Gassem ${ }^{2}$, Anne Lise Bienvenu ${ }^{3}$, Guillaume Bonnot ${ }^{3}$, Stéphane Picot $^{3}$ and Ilhem Mihoubi ${ }^{1}$.}

1. Laboratory of Mycology, Biotechnology and Microbial Activities; Department of Microbiology, Faculty of Natural Sciences and Life, Frères Mentouri University, Constantine (Algeria).

2. Laboratory of Parasitology- Medical Mycology; CHU Ben Badis- Constantine (Algeria).

3. Institut of Parasitology- Medical Mycology, Lyon, SMITH, ICBMS, UMR 5246 CNRS, Claude Bernard- Lyon 1 University (France).

\section{Manuscript Info}

\section{Manuscript History}

Received: 12 July 2016

Final Accepted: 13 August 2016

Published: September 2016

Key words:-

Onychomycosis - Trichophyton rubrum var. raubitschekii - PCR - Algeria.

\section{Abstract}

Onychomycosis is a nail infection caused by different fungal belonging to dermatophytes, yeasts and molds. Dermatophytes are the most incriminated. The aim of our study is to establish both conventional and molecular diagnosis for onychomycosis in Constantine.

Sixteen nail samples were collected from patients with nail lesions, clinically suspected of onychomycosis. Direct microscopic observation and culture on Sabouraud medium with and without cycloheximide were performed. The identification was based on macroscopic and microscopic features, and confirmed by real-time panfungal PCR analysis. The ITS1-5.8S-ITS2 rDNA region was amplified using the ITS1 and ITS4 primers; and sequenced. The 16 samples were positive on their $\mathrm{KOH}$ direct examination. On Sabouraud medium, only 5/16 gave positive culture. The urease test on Christensen medium performed on the 5 isolates revealed a positive urease for one isolate and showed numerous macroconidia in microscopic observation. This isolate showed $99 \%$ of homology with both Trichophyton raubitschekii JX827168.1 and Trichophyton rubrum FM178326.1 in molecular diagnosis. Based on phenotypical characteristics and molecular analysis, this isolate was identified as a Trichophyton rubrum var. raubitschekii. The ITS phylogenetic tree, showed $100 \%$ of homology in the ITS region of Trichophyton rubrum, and Trichophyton raubitschekii isolated. The systematic use of the urease test for Trichophyton rubrum could contribute to increase the prevalence of Trichophyton rubrum var. raubitschekii in the world.

Copy Right, IJAR, 2016,. All rights reserved.

\section{Introduction:-}

Onychomycosis or tinea unguium are the most common causes of nail abnormalities. They represent $50 \%$ of nail diseases with different clinical patterns (Nzenze Afène et al., 2011; Tanrıverdi and Özer, 2013). These infections are

Corresponding Author:- Ilhem Mihoubi. 
mainly caused by dermatophytes, sometimes by yeasts and rarely by environmental molds (Tanrıverdi and Özer, 2013).

Trichophyton rubrum and Trichophyton mentagrophytes are responsible for nearly $90 \%$ of toenail and at least 50\% of fingernail onychomycosis (Tanriverdi and Özer, 2013; Yadav et al., 2015). T. rubrum represents over than 80\% of nail dermatophytes (Gupta and Nakrieko, 2015), and is considered as a complex of species including multiple morphotypes. Recently, several of these morphotypes, have been formally identified as variants of T.rubrum, including T. raubitschekii, usually found in Africa, Southeast Asia, Australia and South America (Hiruma et al., 2012) with tinea corporis and tinea cruris. However, it is weakly associated with tinea pedis and onychomycosis.

Identification of causative agents responsible for onychomycosis is usually based on direct microscopic examination of clinical samples and culture in order to determine the fungal features. The development of molecular biology techniques is a valuable addition to the detection and identification of dermatophytes.

In this context, we used conventional methods (direct examination and culture) and a real-time panfungal PCR assay for the diagnosis of onychomycosis in Constantine (Algeria) in the aim of deciphering T. rubrum complex looking for species closely related to this one.

\section{Patients and methods Nail samples:-}

A total of 16 nails samples were collected from 16 patients oriented by their dermatologists, consulting at the Parasitology- Mycology Laboratory of Constantine Hospital (Algeria) for onychomycosis suspicion. Patients who received a local and/or a systemic antifungal treatment, during the three months before the consultation, were excluded from the study.

Nails were taken from the infected area, in the junction between infected and healthy nail zone (Chabasse and Pihet, 2014).

\section{Clinical sample processing:-}

Each sample was divided into two parts. The first part was treated with $30 \% \mathrm{KOH}$ solution and observed directly under microscope for the presence of fungal elements. The second one was cultured on Sabouraud chloramphenicol medium with and without cycloheximide (Biorad, France) at $27^{\circ} \mathrm{C}$ for up to 4 weeks (Dhib et al., 2014; Nzenze Afène et al., 2011). The identification was based on macroscopic and microscopic features, and confirmed, later, by RT-PCR analysis. The potatoes dextrose agar medium (PDA) was used to stimulate pigmentation and sporulation of the cultures.

\section{Fungal DNA Extraction:-}

DNA extraction was carried out in laboratory of Parasitology and Medical Mycology (Claude Bernard University Lyon 1). DNA was extracted using QiAamp DNA mini kit (Qiagen, Germany) according to the manufacturer's instructions with minor modifications. Small amounts of pure mycelia grown on PDA were transferred in $1.5 \mathrm{ml}$ Eppendorf tubes containing $200 \mu \mathrm{L}$ of physiological water then sonicated during 40 min. twenty $\mu \mathrm{L}$ of proteinase $\mathrm{K}$ and $200 \mu \mathrm{L}$ of buffer ATL were added. Tubes were incubated for 10 minutes at $56^{\circ} \mathrm{C}$ and $200 \mu \mathrm{L}$ of ethanol (96$100 \%$ ) were added. The extracted DNA was recovered in $50 \mu \mathrm{l}$ of Buffer ATE, centrifuged and stored at $-20^{\circ} \mathrm{C}$ until use.

\section{Polymerase chain reaction and DNA Sequencing:-}

The real-time PCR has been realized by Light Cycler according to SYBR Green I technology (Roche Diagnostics Ref.: 2239264), in capillary tubes, using the primers ITS1 (5'-TCCGTAGGTGAACCTGCGG-3) and ITS4 (5'TCCTCCGCTTATTGATATGC-3) (Jackson et al., 1999; Zhang et al., 2015). Real-time PCR technology was performed with fluorescent SYBR Green I. The extracted DNA $(2 \mu \mathrm{L})$ was added to $18 \mu \mathrm{L}$ of reaction mixture containing $10.4 \mu \mathrm{L} \mathrm{H}_{2} \mathrm{O}, 1.6 \mu \mathrm{L} \mathrm{MgCl}_{2}, 2 \mu \mathrm{L}$ of each primer (ITS 1 and ITS 4), $2 \mu \mathrm{L}$ of Light Cycler Fast Start DNA Master SYBR Green I buffer (Roche Applied Science, Meylan, France). Conditions for cycling were $95^{\circ} \mathrm{C}$ for $10 \mathrm{~min}$, followed by 44 cycles of $95^{\circ} \mathrm{C}$ for $10 \mathrm{~s}$ (denaturation), $58^{\circ} \mathrm{C}$ for $10 \mathrm{~s}$ (annealing), and $72{ }^{\circ} \mathrm{C}$ for $40 \mathrm{~s}$ (elongation), followed by a final extension step for $5 \mathrm{~min}$ at $72{ }^{\circ} \mathrm{C}$. Fluorescence was monitored at the end of elongation step.

In order to sequence the fungal DNA, we purified the amplicon using a PCR purification kit (Qiaquick PCR Purification) and sequenced it using ITS1 and ITS4 primers, according to Sanger method. Results were compared on 
a BLAST search via the National Center for Biotechnology Information (NCBI) nucleotide database (http://www.ncbi.nlm.nih.gov/BLAST).

A phylogenetic analysis of sequences representing the Internal Transcribed Spacer (ITS) region of rDNA amplified from T. rubrum isolated, and sequences of T.rubrum complex available in the GenBank database, were constructed with Neighbor joining method (Saitou and Nei, 1987) and with the substitution Kimura two parameters model and a 1000 replicates Bootstrap in Molecular Evolutionary Genetics Analysis- MEGA 6.0 (Kumar et al., 2008). Phylogenetic tree was produced using Trichophyton interdigitale (accession no KP308373.1) as an out-group.

\section{Results:-}

Patients' ages ranged between 23 and 75 years and the sex-ratio was 0,3 . The 16 samples were positive on direct $\mathrm{KOH}$ examination and showed the presence of mycelium (Figure 2). On Sabouraud medium, only 5/16 samples led to positive culture. Patients had two clinical presentations: the distal lateral subungual onychomycosis (DLSO) and the DLSO hyperkeratosis with a thickened nail, brown yellowish color, peeling off at the distal portion of the nail (Figure 1). The cultures were flat to slightly raised, white to cream, sued-like to downy, with a yellow-brown to wine-red reverse. On PDA medium, the five colonies were fluffy disc-shaped with rounded edges and raised centers. Furthermore, they developed wine red pigment on their reverse (Figure 3A and B).
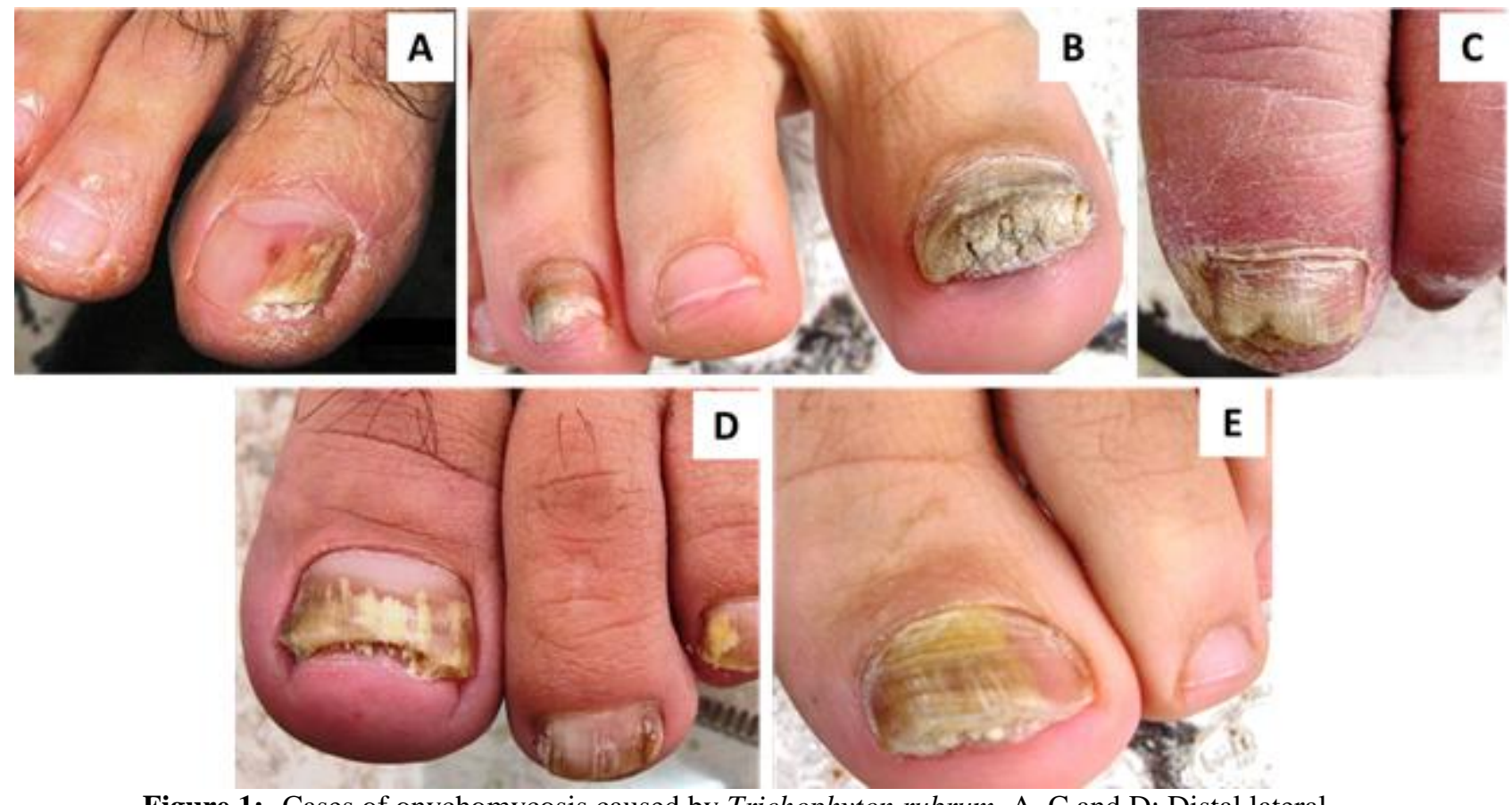

Figure 1:- Cases of onychomycosis caused by Trichophyton rubrum. A, C and D: Distal lateral subungual onychomycosis (DLSO); B and E: DLSO with subungual hyperkeratosis. 


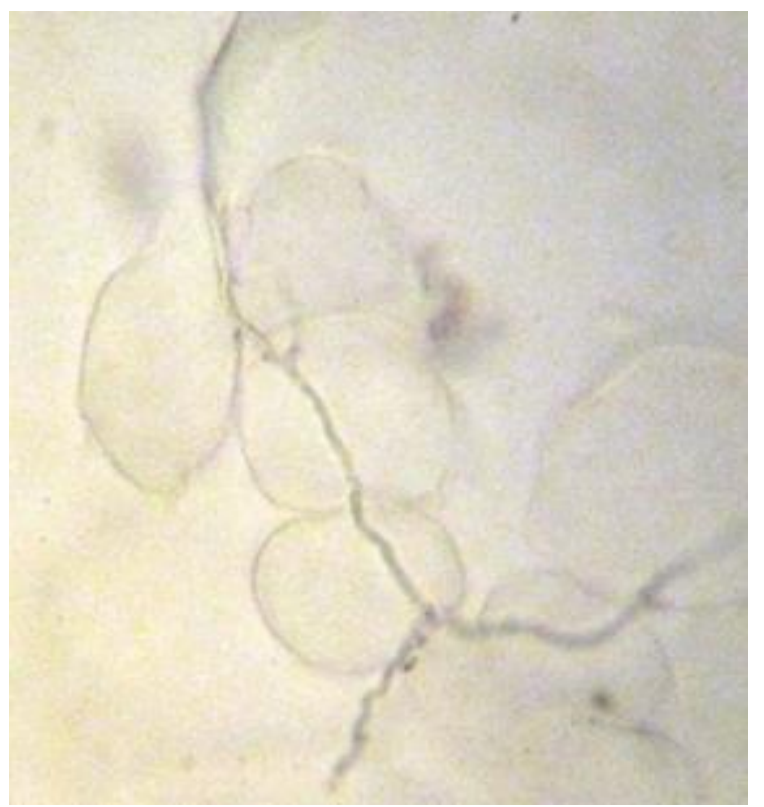

Figure 2:- Positive direct examination of nail scraping showing mycelium.

Microscopically, 4/5 isolates (Tr.1, Tr.2, Tr.3 and Tr.5) produced scanty to moderate number of slender clavate microconidia and no or moderate macroconidia. ITS1 sequence of these isolates was $99 \%$ identical to T.rubrum KC923433.1, AJ270802.1, JX122348.1 and AJ270793.1, respectively. These four isolates were urease negative. One isolate had numerous clavate or pyriform microconidia, abundant production of cylindrical or cigar shaped macroconidia and was urease positive within 6 days of incubation (Figure 3C and D) (Adamski et al., 2014; Arabatzis et al., 2005; Hiruma et al., 2012; Tietz et al., 2002; Zhang et al., 2015). ITS 1 sequence shared $99 \%$ of homology with both T. raubitschekii JX827168.1 deposited by Liu and Zhang in 2012 (China) and T.rubrum FM178326.1. Based on phenotypical characteristics and molecular analysis, we concluded that Tr.4 was a T.rubrum var. raubitschekii. 


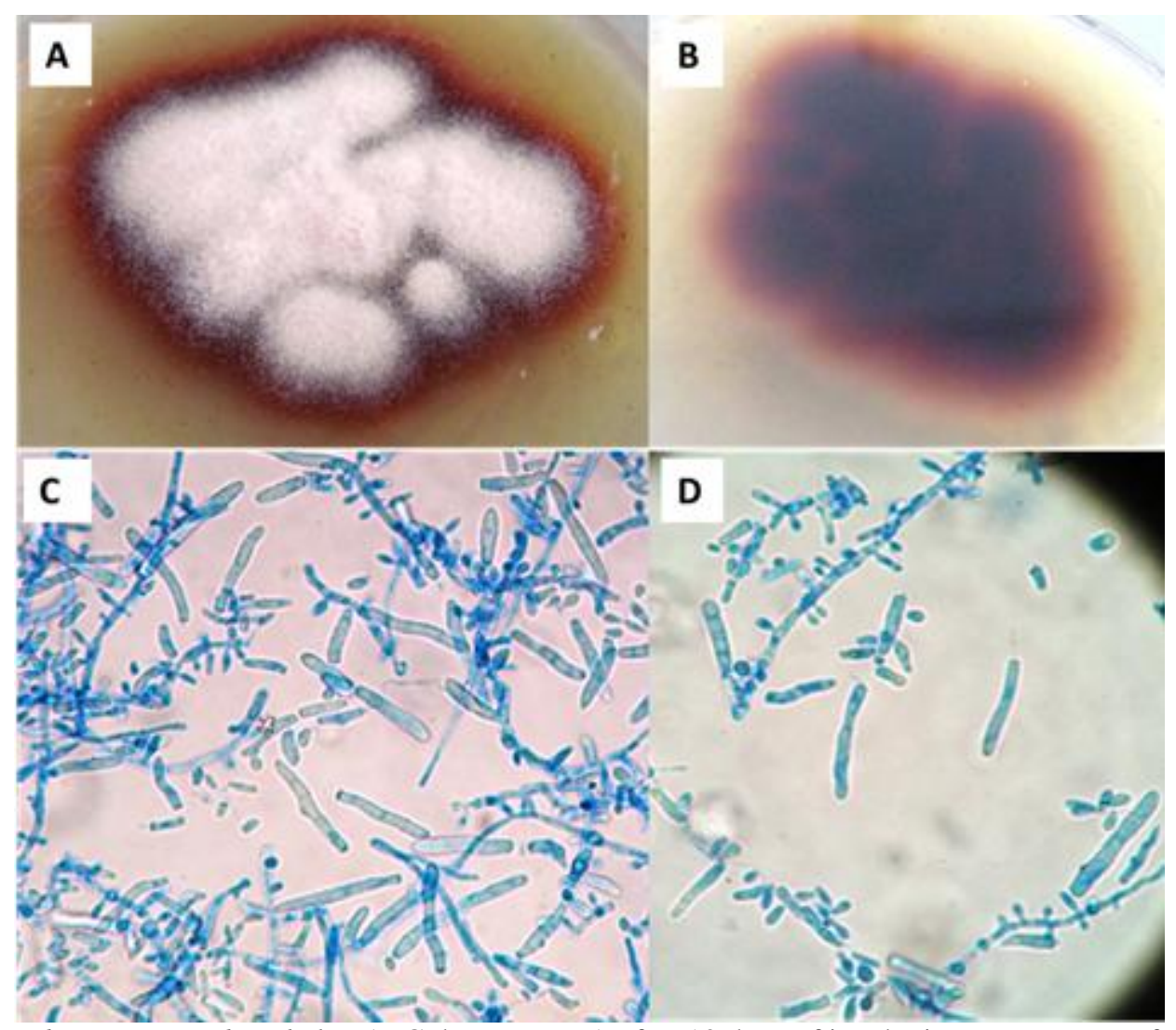

Figure 3:- T. rubrum var. raubitschekii. A: Colony on PDA after 10 days of incubation, B: Reverse of the colony, C and D: Microscopic morphological features X 400.

The ITS phylogenetic tree was composed of two separated clades with T.rubrum and $T$. interdigitale sequences clustering separately. All T.rubrum (Tr.1,Tr.2, Tr.3 and Tr.5), as well as T. raubitschekii (Tr.4), were grouped in the same branch with their related isolates which had 100\% of homology in the 18S_rDNA gene partial sequence ITS1, 5.8S_rDNA gene and ITS2 fragment (Figure 4).

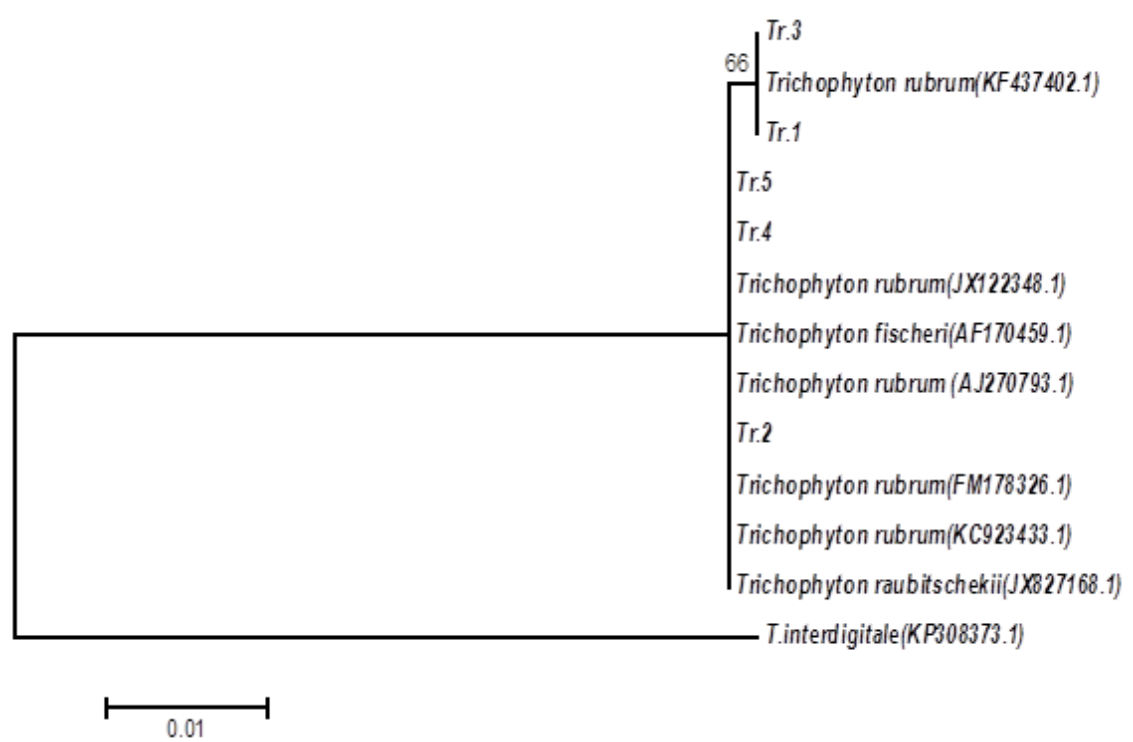

Figure 4:- Phylogenetic tree of T. rubrum, based on confidently aligned rDNA Internal Transcribed Spacer (ITS) sequences. 


\section{Discussion:-}

We reported for the first time T. rubrum var. raubitschekii isolated from the toenail of a hypertensive 69 years old women living in Algeria presenting with an onychomycosis of the hallux. Microscopic examination of cultures and urease positive test were suggestive of T.rubrum var. raubitschekii. The molecular analysis of the isolate led to $99 \%$ of homology with both $T$. raubitschekii JX827168.1 and T. rubrum FM178326.1. As previously reported, we demonstrated that $T$. rubrum var. raubitschekii was genetically identical to T. rubrum. In our study, this variant was isolated in one out of five T. rubrum positive cultures.

T. rubrum var. raubitschekii is an anthropophilic, and pathogenic dermatophyte, originated from Africa, Asia and South America (Brasch, 2007). It was lately found in Europe (Hiruma et al., 2012), but most of the patients involved were immigrants from subtropical and tropical areas. In latest studies, it was associated with tinea corporis, tinea manuum, tinea cruris and tinea unguium (Zhang et al., 2015). Clinically, the lesions caused by T. rubrum var. raubitschekii were indistinguishable from those caused by T. rubrum (Figure 1C). This could explain the fact that no author described this variant before in Algeria. Djeridane et al. reported a global prevalence of toenail onychomycosis of $4.6 \%$ in Algerian military. Onychomycosis were caused by T. rubrum in $35 \%$ of the cases (Djeridane et al., 2006). This could be related to the misidentification of this variant. The colony is phenotypically closed to T.rubrum, granular T.mentagrophytes strains and T. violaceum (van Gelderen de Komaid and Borges de Kestelman, 2001; Zhang et al., 2015). The urease test is not systematically performed in the routine leading to misidentification of T. rubrum var. raubitschekii.

Initially, T. raubitschekii had been classified as a separate species (Adamski et al., 2014) but the molecular studies, such as the chitin synthase gene 1 (CHS1) and internal transcribed spacer (ITS) region (ITS1-5.8S-ITS2) sequences, recognized it as a variant of T.rubrum. Indeed, in our study, we analyzed a ribosomal DNA genomic fragment, consisting of internal transcribed spacer sequences (ITS) 1 and 2 and intermediary 5.8S rDNA, using universal ITS1 and ITS4 primers. We found that T. rubrum var. raubitschekii shares similarities with T. rubrum in sequence of ITS region, suggesting conspecificity as shown on the phylogenetic tree (Figure 4) where all the species are aligned in the same clade. This result can be explained by a genetic homology of both T. rubrum and T. rubrum var. raubitschekii in this genomic region, as previously demonstrated (Gräser et al., 2000). T. rubrum and T.rubrum var. raubitschekii are classified as the same species and considered as synonyms.

\section{Conclusion:-}

Using both conventional and molecular methods, we successfully isolated one T. rubrum var. raubitschekii out of five T. rubrum responsible for onychomycosis in Constantine. Due to genetic homology of the two variants, molecular analysis is not the panacea to differentiate T. rubrum var. raubitschekii and T. rubrum senso stricto. Systematic use of the urease test for $T$. rubrum producing numerous microconidia and macroconidia could contribute to increase the prevalence of T. rubrum var. raubitschekii in the world. To our knowledge, the role of this variant in onychomycosis physiopathology is not elucidated. If T. rubrum var. raubitschekii has a particular pathogenic profile, it could give a rational to implement specific mycological diagnosis to improve its detection.

\section{References:-}

1. Adamski, Z., Kowalczyk, M.J., Adamska, K., Kubisiak-Rzepczyk, H., Bowszyc-Dmochowska, M., Banaszak, A., Bartkiewicz, P. and Zaba, R. (2014): The first non-African case of Trichophyton rubrum var. raubitschekii or a urease-positive Trichophyton rubrum in Central Europe?. Mycopathologia.,178: 91-96.

2. Arabatzis, M., Velegraki, A., Kantardjiev, T., Stavrakieva, V., Rigopoulos, D. and Katsambas, A. (2005): First report on autochthonous urease-positive Trichophyton rubrum (T. raubitschekii) from South-east Europe. Br. J. Dermatol., 153: 178-182.

3. Brasch, J. (2007): Var. raubitschekii of Trichophyton rubrum as a cause of tinea in Germany. Mycoses., 50 Suppl 2: 2-5.

4. Chabasse, D. and Pihet, M. (2014): Mycological diagnosis of onychomycosis. J. Mycol. Médicale., 24: 269278.

5. Dhib, I., Fathallah, A., Yaacoub, A., Hadj Slama, F., Said, M.B. and Zemni, R. (2014): Multiplex PCR assay for the detection of common dermatophyte nail infections. Mycoses.,57: 19-26.

6. Djeridane, A., Djeridane, Y. and Ammar-Khodja, A. (2006): Epidemiological and aetiological study on tinea pedis and onychomycosis in Algeria. Mycoses., 49: 190-196. 
7. Van Gelderen de Komaid, A. and Borges de Kestelman, I. (2001): Urease-positive Trichophyton rubrum strains (previously described as T. raubitschekii): first isolations in Argentina. Eur. J. Epidemiol.,17: 929-933.

8. Gräser, Y., Kuijpers, A.F.A., Presber, W. and de Hoog, G.S. (2000): Molecular Taxonomy of the Trichophyton rubrum Complex. J. Clin. Microbiol., 38: 3329-3336.

9. Gupta, A.k. and Nakrieko K.A. (2015): Trichophyton rubrum DNA strain switching increases in patients with onychomycosis failing antifungal treatments. Br. J. Dermatol., 172: 74-80.

10. Hiruma, M., Kano, R., Sugita, T., Mochizuki, T., Hasegawa, A. and Hiruma, M. (2012): Epidemiological aspects of Trichophyton rubrum var. raubitschekii in Japan. J. Dermatol., 39: 1000-1001.

11. Jackson, C.J., Barton, R.C. and Evans, E.G. (1999). Species identification and strain differentiation of dermatophyte fungi by analysis of ribosomal-DNA intergenic spacer regions. J. Clin. Microbiol., 37: 931-936.

12. Kumar, S., Nei, M., Dudley, J. and Tamura K. (2008): MEGA: a biologist-centric software for evolutionary analysis of DNA and protein sequences. Brief Bioinform., 9: 299-306.

13. Nzenze Afène, S., Ngoungou, E.B., Mabika Mamfoumbi, M., Bouyou Akotet, M.K., Avome Mba, I.M. and Kombila, M. (2011): Les onychomycoses au Gabon : aspects cliniques et mycologiques. J. Mycol. Médicale. J. Med. Mycol., 21: 248-255.

14. Saitou, N. and Nei, M. (1987). The neighbor-joining method: a new method for reconstructing phylogenetic trees. Mol. Biol. Evol., 4: 406-425.

15. Tanriverdi, S.T. and Özer, Ö. (2013): Novel topical formulations of Terbinafine-HCl for treatment of onychomycosis. Eur. J. Pharm. Sci., 48: 628-636.

16. Tietz, H.J., Hopp, M. and Gräser, Y. (2002): First isolation of Trichophyton raubitschekii (syn. T. rubrum) in Europe. Mycoses., 45: 10-14.

17. Yadav, P., Singal, A., Pandhi, D. and Das, S. (2015): Clinico-Mycological Study of Dermatophyte Toenail Onychomycosis in New Delhi, India. Indian. J. Dermatol., 60: 153-158.

18. Zhang, H., Xiong, X., Liu, T. and Ran, Y. (2015): Generalized superficial mycosis caused by Trichophyton raubitschekii in China: case report and review of the literature. Mycopathologia., 179: 279-284. 